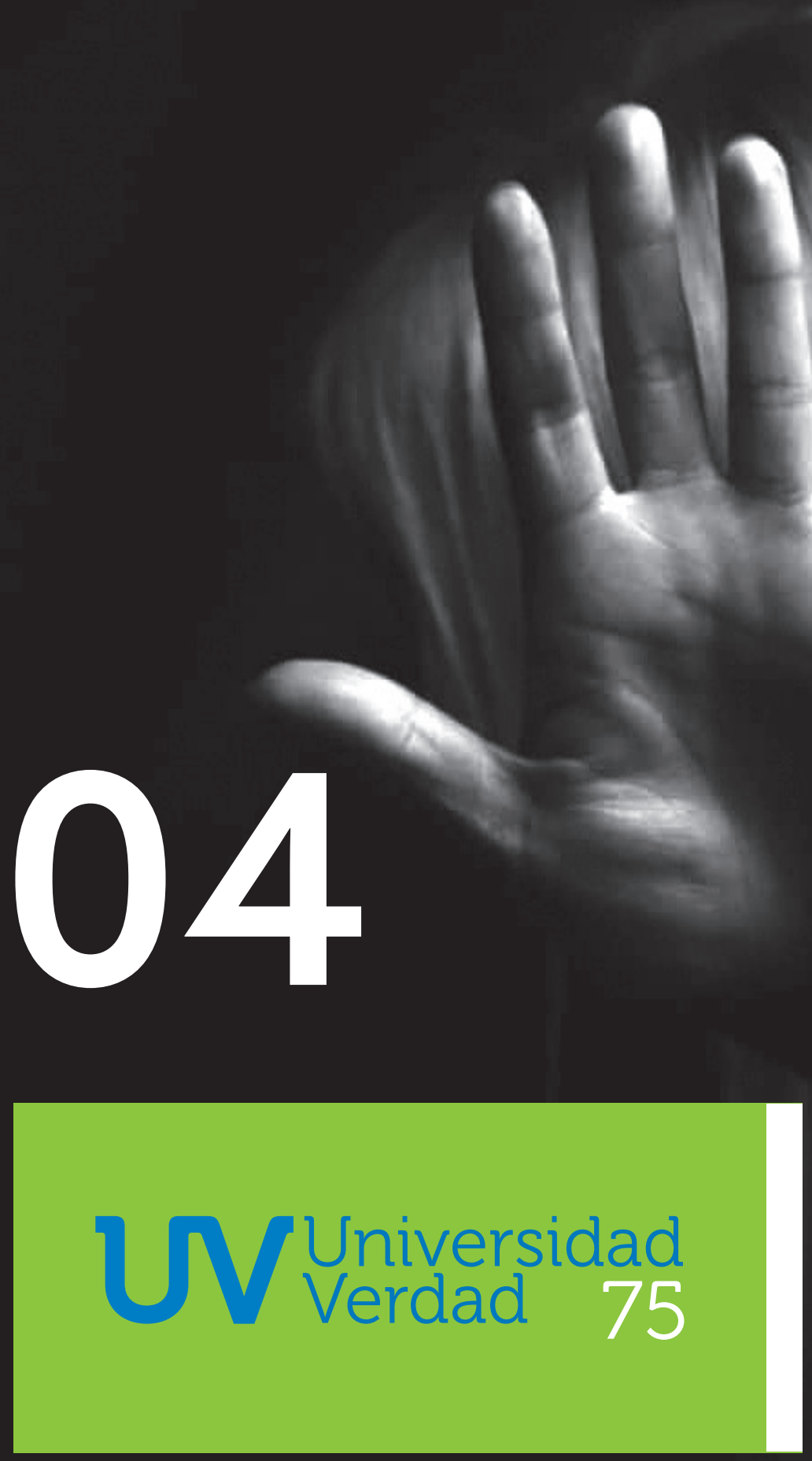




\section{CONTINUIDADES COLONIALES: DEL DISCURSO DE LA PROTECCIÓN A LA FAMILIA A LA REGULACIÓN DE LA VIOLENCIA CONTRA LAS MUJERES EN EL DERECHO ECUATORIANO DEL SIGLO XX}

\section{Colonial continuities: from the discourse of family protection, to the regulation of violence against women in Ecuadorian twentieth century law}

Dra. Silvana Tapia Tapia. Profesora Titular de la Facultad de Ciencias Jurídicas. Universidad del Azuay. UDA. (Ecuador) (stapia@uazuay.edu.ec) (https://orcid. org/0000-0002-5598-7098)

\begin{abstract}
Resumen
Este artículo examina momentos claves de la historia de la legislación ecuatoriana sobre la violencia contra las mujeres en el siglo XX. Revela cómo las construcciones coloniales y poscoloniales de la femineidad han atravesado al derecho y a los discursos jurídicos durante el siglo pasado. El ideal europeo de la mujer-madre, cristiana, blanco-mestiza, de clase media-alta, sexualmente recatada, se introdujo a través de la colonización y se consolidó después de la independencia, emergiendo como eje crucial para la construcción de un Estado-nación unitario, a través de la protección legal a la familia. La producción de lo femenino como inextricablemente ligado a la procreación y a la crianza, por una parte, facilitó la recepción estatal de las propuestas de los movimientos de mujeres para combatir la violencia contra las mujeres hacia finales del siglo XX, pero al mismo tiempo produjo una representación de la violencia contra las mujeres como un atentado contra la armonía
\end{abstract}

familiar. Esto genera el riesgo de excluir de la esfera de protección del derecho a personas cuyos cuerpos racializados, engenerizados y precarizados no corresponden al paradigma de la mujer de familia digna de ser protegida, riesgo de muy probable proyección hacia las leyes sobre violencia de género del siglo XXI.

\section{Abstract}

This paper examines key moments in the history of Ecuadorian legislation on violence against women during the 2oth century. It reveals how colonial and postcolonial constructions of femininity have crossed historical law and juridical discourses. The European ideal of the woman-mother, Christian, White-mestiza, of upper middle class, sexually contained, was introduced through colonisation and consolidated after the Independence, emerging as a crucial axis for the construction of the unitary Nation-State, through the legal protection of the family. The production of the feminine as inextricably linked to 
reproduction and child-rearing, on the one hand, facilitated the state reception of women's movements' proposals to combat VAW by the end of the 2oth century; but, on the other hand, it produced a representation of VAW as an attack against family harmony. This runs the risk of excluding from the scope of legal protection those persons whose racialised, engendered and precarious bodies do not correspond to the paradigm of the family woman who deserves protection. This is likely projected into the VAW legislation of the 21st century.

\section{Palabras clave}

Violencia contra las mujeres, Ecuador, feminismo decolonial, violencia familiar, siglo veinte.

\section{Keywords}

Violence against women, Ecuador, decolonial feminism, family violence, twentieth century.

"A diferencia de la colonización, la colonialidad del género todavía está con nosotros; es lo que yace en la intersección entre género/clase/raza como constructos centrales del sistema mundial capitalista de poder". (Lugones, 2010)

\section{1.}

Introducción. Este artículo explora cómo las estrategias legales y de política pública sobre la violencia contra las mujeres durante el siglo XX fueron vehículos de narrativas instituidas a través de la colonización acerca de las mujeres dentro de la familia. Se analiza cómo los discursos jurídicos han reproducido la colonialidad del género - concepto acuñado por feministas decoloniales- pese a que las reformas legales usualmente se presentan como un avance en materia de derechos de las mujeres. Como consecuencia, la investigación arroja luz sobre por qué las demandas de reforma legal en materia de violencia contra las mujeres han sido típicamente más exitosas que otras propuestas, como la despenalización del aborto, o la protección a las trabajadoras sexuales y las mujeres trans, que no han tenido visibilidad.
La literatura feminista crítica sobre justicia penal (Bernstein, 2012; Halley, Kotiswaran, Shamir, \& Thomas, 2006; Halley, Kotiswaran, Shamir, \& Rebouché, 2018) ha identificado el fortalecimiento de la llamada "gobernanza feminista" a nivel global, y ha señalado que una versión de ésta se ha traducido en un "feminismo carcelario" asociado con la expansión de la penalidad y el control social. Estos a su vez derivan de la expansión del neoliberalismo y el declive del Estado de bienestar. La nueva gobernanza global también ha difundido una representación medicalizada y tecnificada de la violencia contra las mujeres, que junto con la noción liberal de la responsabilidad individual, han contribuido al posicionamiento del derecho penal y otras formas de control social como instrumentos adecuados para proteger derechos fundamentales (Bernstein, 2012; Bumiller, 2008; Corrigan, 2006; Gotell, 1998; Halley et al., 2006).

Los mencionados análisis sin duda contribuyen a la comprensión de un problema contemporáneo complejo, pero se refieren en su mayoría a países del norte global, por lo que quedan preguntas pendientes respecto a sitios poscoloniales donde muchas mujeres sufren opresiones superpuestas por cuestiones no solo de género, sino de clase y raza, las cuales son sintomáticas de la marginación de los cuerpos colonizados, racializados y engenerizados. Consideremos, por ejemplo, que mientras la idea de proteger a las madres de familia de la violencia no ha sido tan difícil de introducir en el derecho, rara vez se han priorizado las necesidades específicas de grupos en graves condiciones de vulnerabilidad, como las trabajadoras sexuales, las mujeres campesinas, indígenas y afrodescen- 
dientes, y las mujeres trans, por nombrar algunos ejemplos. Entonces, es importante reconocer la posibilidad de que haya narrativas coloniales con impacto continuado en las políticas públicas de erradicación de la violencia contra las mujeres.

Los hallazgos de este artículo sugieren que las lógicas discriminatorias subyacentes a los discursos sobre violencia contra las mujeres en el siglo XX no han prosperado solo a través del neoliberalismo, la gobernanza global y el llamado "feminismo carcelario". Muchos discursos que han sostenido los procesos legislativos relacionados con violencia de género ya estuvieron racionalizados e integrados en construcciones coloniales de la femineidad y la familia en América Latina. El ideal europeo de la mujer-madre, cristiana, blanco-mestiza, de clase media-alta, sexualmente recatada, se consolidó a través de la colonización y se fortaleció después de la independencia, con la figura de la madre que cría buenos ciudadanos como un eje para la construcción del nuevo Estado-nación. Esta narrativa se ha reproducido de forma aparentemente benigna en el discurso legal del siglo XX, pues ha facilitado la aprobación de leyes diseñadas para proteger a las mujeres. No obstante, este artículo muestra que la representación colonial del cuerpo femenino prosperó durante el siglo pasado, y que los marcos discursivos más amplios sobre el rol de las mujeres y los requisitos que ellas deben cumplir para ser consideradas sujeto de derechos, no han sido radicalmente cuestionados. Los hallazgos aquí expuestos nos ayudan a proyectar un marco analítico para evaluar a futuro cómo varios caracteres de las leyes del siglo XXI pueden también ser resultado de la colonialidad del poder y del género, pese a que las leyes se perciben como políticamente neutrales, incluso por parte de los movimientos sociales. Cabe, pues, repensar a la reforma legal como instrumento para el proyecto feminista emancipatorio.

\section{a. Metodología}

La investigación empleó una metodología cualitativa socio-legal para examinar las construcciones discursivas en las que se fundamentaron las leyes estudiadas. A diferencia de la investigación jurídica tradicional, esta metodología interdisciplinaria tiene en cuenta los significados sociales de las instituciones jurídicas y las relaciones de poder que se despliegan a través del derecho (Bacchi, 1999; Shore \& Wright, 1997). Los hallazgos están basados en información obtenida a través de trabajo de campo que incorporó fuentes primarias múltiples. Éstas incluyeron evidencia documental y testimonial. El material documental comprendió una base de datos histórica de la legislación ecuatoriana desde del siglo $\mathrm{XX}$, a la que se sumaron textos de archivo escritos por mujeres feministas, contextualizados a través del trabajo de historiadoras que tratan a América Latina como un sitio postcolonial. Además, se examinaron los registros de debates parlamentarios sobre la ley 103 y las reformas penales de 2005, obtenidos del archivo de la Asamblea Nacional, así como documentos institucionales de agencias estatales y organizaciones no gubernamentales que intervinieron en la regulación de la violencia contra las mujeres a partir de la década de 1980.

Para el análisis de las décadas de 1970 y 1980, se incorporaron historias orales narradas por protagonistas históricas del movimiento de mujeres del Ecuador: en 2015 se llevaron a cabo 25 entrevistas semiestructuradas con personas autoidentificadas como feministas, incluyendo exlegisladoras, exfuncionarias públicas y profesionales independientes. Todos los nombres se han anonimizado.

Para el análisis de todos los materiales se empleó una modalidad inspirada por las intervenciones metodológicas de Foucault - arqueología y genealogía-, y se aplicaron métodos feministas de análisis de discurso (Bacchi, 1999, 2012). La información se codificó utilizando software de análisis de datos cualitativos.

Cabe reconocer que debido a que el artículo versa sobre leyes y políticas públicas, incorpora principalmente las voces de mujeres que consiguieron en alguna medida ser escuchadas por las instituciones de gobernanza. Estos grupos están mayoritariamente compuestos por personas mestizas y de clase media. Otros grupos comunitarios, indígenas, rurales, afro-descendientes, etc., han cumplido un papel central en las luchas emancipatorias de las mujeres en el Ecuador, pero no han accedido regularmente a los espacios desde los que se genera el derecho.

\section{2. El derecho como tecnología de gubernamentalidad y vehículo de la colonialidad. Si bien América Lati- na suele considerarse parte del mundo occiden- tal, en muchos aspectos no lo es. Con un largo}


pasado colonial, el subcontinente está habitado por mestizos, afrodescendientes y pueblos indígenas, que resistieron la opresión colonial y han mantenido vivas sus tradiciones, cosmovisiones y formas comunitarias de organización, las cuales están presentes en los debates políticos contemporáneos. Luego de las guerras de independencia, las clases gobernantes en América Latina enfrentaron el desafío de construir estados en espacios profundamente heterogéneos, donde las estructuras sociales coloniales se mantuvieron vigentes, pero también fueron desafiadas, no solo por los movimientos de base, sino también por las elites competidoras, mientras se buscaba construir una identidad nacional para hacer más gobernables a las nuevas repúblicas.

El derecho y las políticas públicas cumplieron un rol crucial en la construcción de los ideales sobre los que se pretendía fundar una nación homogeneizada: a través de mecanismos legales se regularon instituciones como las condiciones para el goce de la ciudadanía y la implementación de la democracia (Padrón, 2019), al tiempo que se desarrollaron estrategias de política pública para manejar a la población. Éstas incluyeron la regulación de los roles de género. Tal es el caso del discurso del cuidado de la niñez durante la primera mitad del siglo XX, como un deber patriótico de las mujeres ecuatorianas, consistente en criar buenos ciudadanos (Clark, 2001). Para entender las políticas de manejo de la población, es útil el concepto foucaultiano de "gubernamentalidad" (Foucault, 1991; Oksala, 2013), entendido como la disposición móvil de maneras de pensar relacionadas con el gobierno óptimo de las poblaciones, donde las "prácticas divisorias", es decir las clasificaciones de los sujetos, de los campos del conocimiento, de lo "normal" y "desviado", etc., producen estándares que pretenden asegurar la cohesión social y la optimización de la población, y por lo tanto informan a las estrategias de gobernanza.

Adicionalmente, las ideas de Foucault respecto a la producción de la subjetividad (en tanto concepto filosófico) y de "regímenes de verdad" a través de las interacciones entre el poder y el saber (Foucault, 1982), permiten abordar la subjetividad legal femenina y comprender mejor la interdependencia entre los regímenes de disciplinamiento y regulación de la población, y los sistemas legales (Golder \& Fitzpatrick, 2009). Por ejemplo, basándose en el trabajo de Foucault sobre la idea de raza, ${ }^{1}$ Ann Stoler (1995) ha rastreado varias conexiones entre las prácticas coloniales y la formación de las subjetividades de género racializadas, que ella considera centrales para el "orden colonial de las cosas". Éste último concepto nos permite conectar el trabajo de Stoler con el de las feministas latinoamericanas, que consideran que el orden colonial está fundamentado en la creación de las categorías separables y binarias propias de la modernidad, donde la distinción entre cuerpos humanos y no humanos es el núcleo del proyecto colonial. Así, María Lugones (2010) acuñó la noción de "colonialidad del género" con el objetivo de expresar la intersección entre raza, clase y género en las sociedades poscoloniales.

Vistas a la luz de estos marcos teóricos, las estrategias de optimización de la población que las jóvenes repúblicas latinoamericanas implementaron, no desafiaron, sino que reprodujeron los ideales europeizados de familia, nación y femineidad. Autores como Aníbal Quijano (2000) y Walter Mignolo (2011) se han referido a las teorías y prácticas derivadas de la "colonialidad del poder". Según esta perspectiva, las jerarquías dicotómicas de lo humano y lo no humano, es decir, la "diferencia colonial" marcada por la racialización, es un elemento constitutivo de los patrones de poder capitalistas globales. La colonialidad, entonces, es una negación persistente de los modos de ser que no se ajustan a los paradigmas europeos. Las feministas decoloniales han completado la noción de colonialidad del poder con la de colonialidad del género, y han sostenido que las luchas políticas en sitios poscoloniales han producido la negación de las cosmovisiones no occidentales y la imposición de los binarios y jerarquías de género que caracterizan al pensamiento occidental.

De acuerdo con las perspectivas feministas decoloniales, la heteronormatividad colonial impuso un ordenamiento jerárquico que implantó un modelo por el cual lo femenino complementa a lo

1. Stoler señala que el tema de la producción de la raza es más importante en el trabajo de Foucault de lo que generalmente se ha reconocido 
masculino poniéndose a su servicio: las mujeres atienden a los hombres, se quedan en casa, cuidan a sus hijos, etc. (Gargallo, 2014). En otras palabras, la colonialidad del género puede producir roles de género fijos y jerárquicos que se reproducen en las regulaciones legales. Entonces, la colonialidad y la ley desempeñan un papel central en la descalificación de los "otros" y en la estandarización de las experiencias de vida de las subjetividades subordinadas (Quijano, 2000).

El marco conceptual de la colonialidad del género es, pues, adecuado para comprender la producción dicotómica del sujeto y de los cuerpos racializados y engenerizados como entidades desviadas que necesitan reforma y "blanqueamiento"; por lo que la "rectificación" de dichos cuerpos se vuelve, históricamente, un fin central de la ley y de la política pública en los espacios poscoloniales. La noción de "colonialidad del género" permite comprender la opresión de las mujeres como una interacción compleja de los sistemas económico, racial y de género.

Volviendo a la noción de gubernamentalidad, si bien el feminismo decolonial latinoamericano no ha incorporado directamente conceptos foucaultianos, es útil el ejercicio analítico de leer la gubernamentalidad a la luz de las teorías decoloniales. Los conceptos de subjetivación y la gubernamentalización nos muestran cómo los regímenes disciplinarios usados para gobernar producen cuerpos dóciles inscritos en la historia. Esto, mirado a través del lente decolonial, permite ver que el cuerpo femenino en América Latina se hace inteligible dentro de los parámetros de la familia cristiana, blanca y heteronormativa, que es un sitio nuclear de normalización para el discurso del fortalecimiento de la nación, como se explica a continuación.

\section{3.}

\section{La protección}

\section{a la familia en la primera}

mitad del siglo XX. Esta sección revela las conexiones históricas entre las representaciones poscoloniales de la familia y las respuestas estatales a la violencia doméstica desde inicios del siglo XX en el Ecuador. Según lo han mostrado varias historiadoras feministas (Dore \& Molyneux, 2000; Guy, 2000; Tinsman, 2001), la construcción de las mujeres como sujetos de protección legal en América Latina ha estado vinculada con la protección a la familia como un objetivo fundamental de los estados con respecto a la consolidación de la identidad nacional. Después de las guerras de independencia del siglo XIX, las elites gobernantes mestizas y blancas enfrentaron la tarea monumental de reunir a pueblos y comunidades heterogéneas para formar las nuevas repúblicas. A pesar de su respaldo retórico al liberalismo y a las nociones de igualdad ante la ley, las elites gobernantes no desafiaron en la práctica las jerarquías sociales que había producido la colonización, sino que tendieron a "denigrar lo local y valorar a Occidente" (Radcliffe \& Westwood, 1996, p. 13). Distinciones como la casta, la profesión y el apellido, que habían estructurado la sociedad antes de la introducción de las ideas liberales en el siglo XVIII, continuaron siendo fundamentales para la vida política en los años republicanos (Guy, 2000). La familia arquetípica, articulada a través de paradigmas aristocráticos, blancos y heterosexuales, sirvió como una el eje alrededor del cual se buscó formar la identidad nacional y la ciudadanía óptima. Las clases dominantes vieron a la familia como una institución que podría dar a los nuevos territorios independientes la estabilidad necesaria para construir un Estado-nación fuerte.

Desde los estudios de la gubernamentalidad, la optimización de la población se ha revelado como una meta que pretende alcanzarse modificando los comportamientos de los individuos "desviados". En América Latina, la colonialidad ha dado como resultado una asociación entre la desviación, la raza y el género. Así, por ejemplo, existió una proliferación de discursos sobre la salud de la "raza nacional" que se remonta a la familia como un espacio para corregir a los ciudadanos. En la región, la idea de raza se entendió más en relación con la conducta que con las características estrictamente fenotípicas de la población, con fundamento en la noción neolamarckiana de transmisión hereditaria de caracteres adquiridos, por oposición a la eugenesia darwiniana (Stepan, 1991). Un claro ejemplo son las políticas públicas basadas en la idea de los "venenos raciales" (ver Ilustración 1), un término utilizado a principios del siglo XX para describir la "contaminación racial" a través de fluidos corporales, que se creía transmitía males como el alcoholismo y las enfermedades venéreas, llegan- 
do incluso a prohibirse, en países como Chile, el amamantamiento por nodrizas de raza "inferior" (Clark, 2001; Stepan, 1991).

\section{Ilustración 1. Facsímil de un artículo científico sobre "venenos raciales", de 1910}

\section{RACIAL POISONS}

\section{ALCOHOL}

By C. W. SAlebby, M.D., F.R.S.E., F.Z.S.

\section{A. Preliminary Considerations}

IT may be as well to begin by removing a misconception regarding the use of the term racial poison. Unfortunately it has been employed in various quarters lately as if it were applicable to any substance that poisons a large number of individuals at any time. When thus used, however, the differentia of the term is entirely missed. Indeed, the term was introduced by the present writer some three years agb precisely in order to distinguish between the action of substances upon an individual as an individual and their action through the individual upon the race: " by a racial poison I understand a substance which, whether or not injuring the individual who takes it, is liable to injure the race of which he [or she] is the trustee." It is allimportant for the eugenist to make this distinction. Thus strychnine is a poison, but deadly though it be to the individual, it is so far as we know not a racial poison-except in so far as that anything which kills the individual interferes with the race. Again, the community as a whole takes very many poisons-as, for instance, boric acid-in its food every day, and this is a question by no means negligible; but it is a problem in hygiene, not in eugenics, so long as we are without any evidence that these adulterants prejudice the individual's parenthood. Morphine, on the other hand, is believed by the most authoritative observers, such as the late Professor Brouardel, ${ }^{1}$ to have a racial influence, and a still more recent student of high authority, Dr. Harrington Sainsbury, writes as follows:

1 Opiuw, Morphine, it Cocaine, P. Brouardel (Baillière ot fils, 1906), p. 68.

El derecho jugó un papel importante para el propósito de optimizar a la ciudadanía: los Estados latinoamericanos invirtieron en las funciones retóricas y educativas de la ley, preservando la tradición platónica por la cual el derecho es crucial para educar y producir ciudadanos "virtuosos" (Htun, 2003). El impulso para administrar a la familia como núcleo de la sociedad se desplegó a través de la regulación civil de la propiedad, la custodia de los hijos, la capacidad legal de las mujeres y el régimen del matrimonio y el divorcio. Asimismo, se establecieron disposiciones penales que criminalizaban la "desviación" a través de categorías como el adulterio, la prostitución, el aborto, la homosexualidad y el infanticidio, con el fin moral subyacente de preservar la armonía y la cohesión de la familia heteronormativa. A menudo, las decisiones judiciales penales servían para castigar a las mujeres que no se ajustaban a los ideales burgueses de la moral doméstica (Díaz, 2001).

Un ejemplo claro es la regulación del adulterio del Código penal ecuatoriano de 1906: "Art. 378. - La mujer convencida de adulterio, será condenada a prisión de tres a cinco años. El marido puede suspender el efecto de esta condena, consintiendo en volver a tomar a su mujer" ${ }^{1}$.Desde la promulgación del primer Código Penal ecuatoriano, el adulterio fue un delito que sólo podía ser cometido por las mujeres. No había una categoría equivalente para los hombres, aunque a partir de 1938 se introdujo como delito la convivencia continua de un marido con una concubina (el llamado "amancebamiento"). En otras palabras, la sexualidad de las mujeres se vigilaba en todo momento, mientras que la de los hombres sólo se penaba si representaba una interrupción severa de la armonía familiar.

Así, se esperaba que la legislación ayudara a "blanquear" y "civilizar" a la población a través de la modificación de sus hábitos. Las mujeres fueron incorporadas a estos proyectos principalmente con su responsabilización por el desarrollo de la niñez, entendida como un deber patriótico que debía obedecer al conocimiento médico y científico de la época, para criar buenos ciudadanos (Clark, 2001; Guy, 2000; Rendón, 2006). La convergencia entre los ideales nacionales y los deberes maternos facilitó la articulación de la idea de que era necesario ayudar y proteger a

2. El adulterio fue despenalizado en 1983 siguiendo las sugerencias de eruditos liberales de la época, quienes consideraron que la disposición era arcaica. El adulterio sigue siendo un fundamento legal para demandar el divorcio según el Código Civil en vigor. 
las madres para modernizar a la nación y asegurar la educación adecuada de los niños. Bajo tales lógicas, se diseñaron leyes para intervenir en la familia y neutralizar a los miembros violentos de la misma.

Un factor que facilitó el desarrollo de estas leyes, fue la existencia de mecanismos legales de intervención en las disputas conyugales que se usaban desde el período colonial y se extendieron hacia el período republicano (Dore, 2000; Rodríguez, 2000). Estos eran generalmente procedimientos de derecho privado, pero a menudo habilitaban a las mujeres a solicitar que sus parejas abusivas fueran enviadas a casas correccionales u hospicios. Un ejemplo afín se puede encontrar en la legislación penal ecuatoriana de los años treinta. En medio del entusiasmo por la eugenesia, la higiene pública y la intervención científica en la familia, el discurso estatal enmarcó al alcoholismo como un "veneno racial" que ponía en peligro la construcción de la nación (Clark, 2001; Stepan, 1991). La regulación del alcoholismo a su vez ofreció a las mujeres herramientas legales para aliviar en algo el abuso doméstico que sufrían, lo que constituye un caso de uso temprano del sistema penal como medio para mitigar la violencia familiar: el Código Penal de 1938, promulgado durante una dictadura militar, contenía la siguiente disposición sobre el alcoholismo crónico:

Art. 607.- A los que dentro de un término de noventa días hubieren reincidido en embriaguez por cuatro veces, se les destinará a una casa de temperancia, u otro lugar a propósito, para que permanezcan en ella por un tiempo de seis meses a dos años; pudiendo ampliarse o restringirse este plazo, y aun revocarse la detención, cuando el detenido estudio del intimado dé suficientes pruebas de haberse reformado. (Jefatura Suprema del Ecuador, 1938)

El mejor argumento para que las mujeres pudieran convencer a la policía de arrestar a los hombres bajo esta disposición, como lo ha confirmado Clark (2001), era argüir que se habían vuelto peligrosos para sus familias debido a la agresividad causada por la embriaguez crónica. Naturalmente, esta disposición no fue el resultado de propuestas feministas; estaba más bien inscrita en discursos estatales de optimización de la nación en los cua- les el poder patriarcal no fue cuestionado, sino que se reprodujo de una manera sutil. El citado artículo revela una convergencia entre varias narrativas en torno al problema de la violencia doméstica: subyacen a esta regulación estrategias para "mejorar" la raza nacional purificando el comportamiento individual. La embriaguez como veneno racial, junto a la agresividad e irresponsabilidad de los jefes de hogar se consideraron formas de "desviación" a las que la ley debía dar respuesta. Las madres correctas y obedientes eran quienes caían dentro del ámbito de protección de la norma. Las conductas que se consideraban características de la inferioridad racial, y por lo tanto moral, debían ser modificadas para "blanquear" a la población. En otras palabras, los varones podían ser sancionados si se desviaban de sus deberes como padres proveedores (Tinsman, 2002), porque tal desviación era considerada peligrosa para la familia y, en consecuencia, para la nación.

Como vemos, el derecho puede funcionar como una tecnología gubernamental que, además de cumplir su papel en el control social y la vigilancia, hizo las veces de aparato de normalización destinado a alentar comportamientos "civilizados". Los mecanismos legales que dieron a las mujeres acceso a herramientas para aliviar la violencia coexistían con disposiciones legales que las criminalizaban (como el adulterio, el concubinato, la prostitución, el aborto, el infanticidio, etc.), así como con leyes que aprobaban o reducían las sanciones para los hombres que cometían delitos como el de matar a una esposa infiel, a una hija promiscua u otras familiares "desviadas". El siguiente ejemplo es un artículo tomado del Código Penal de 1938:

Art. 22.- Tampoco hay infracción alguna cuando uno de los cónyuges mata, hiere o golpea al otro, o al correo, en el instante de sorprenderlos en flagrante adulterio, o cuando una mujer comete los mismos actos en defensa de su pudor, gravemente amenazado.

Puesto que, como se explicó previamente, el adulterio solo podía ser perpetrado por mujeres, el artículo prácticamente autorizaba el asesinato de las esposas infieles. Otro ejemplo viene dado por la siguiente disposición, que estuvo vigente hasta 2005: "Art. 509.- Llámase estupro la cópula con una mujer honesta, empleando la seducción o enga- 
ño para alcanzar su consentimiento (énfasis agregado por la autora)." Se colige que solo las mujeres "honestas" podían ser víctimas de estupro, lo que excluía implícitamente a las mujeres "desviadas" (como las trabajadoras sexuales, las madres solteras, etc.) del ámbito de protección de la ley, ya que no habrían sido consideradas "honestas".

La subordinación de las mujeres a través del derecho fue uno de los principales objetos de crítica de los movimientos feministas de la época: así, Marena Briones, abogada guayaquileña, escribió varios artículos en la década de 1980 afirmando que las leyes exponían el estatus de las mujeres como ciudadanas de segunda clase (Briones, 1988). Briones denunció enfáticamente los fundamentos morales de la regulación penal de la sexualidad de las mujeres, que protegía valores como la castidad y la virginidad, y expuso a la familia como el "origen de la opresión de las mujeres, porque ha sido hasta hoy una institución patriarcal que ha glorificado la reproducción de la especie, y la ley ha consolidado las desigualdades" (1988, p.4). En vista de que solo las mujeres circunscritas a los límites de la vida de familia se consideraban dignas de protección legal, sus necesidades de protección cedían frente a las de la familia, lo que dio como resultado una equiparación entre las dos.

Se han mostrado, pues aparatos discursivos legales que legitimaban la protección de las mujeres en tanto madres de familia "correctas". Estos discursos jurídicos son preexistentes a los discursos de protección de las mujeres basados en derechos humanos, como los que habrían de prevalecer hacia finales del siglo XX. De esta manera, según se verá a continuación, la oleada de leyes sobre la violencia contra las mujeres en la década de 1990 sería bien recibida, debido a su anclaje en el discurso de la "violencia contra la mujer y la familia".

\section{4 . \\ El boom de la década de 1990 en la legislación sobre la violencia contra las mujeres. Este acápite muestra cómo la continuidad de las narrativas poscolo- niales sobre la protección a la familia facilitó la recepción de las propuestas de los movimientos de mujeres en la década de 1990, modulándolas}

al mismo tiempo. También se evidenciará la incorporación del marco punitivo que persiste en la actualidad, proceso en el cual la acción de la legislatura de la época fue clave, anteponiéndose en muchos casos a los encuadres propuestos por los movimientos de mujeres.

El surgimiento de movimientos de mujeres institucionalizados en América Latina, generalmente se sitúa en la transición a la democracia, después de las dictaduras militares (UNESCO, 1984). En el Ecuador, esto ocurrió a fines de la década de 1970, con el establecimiento de las las primeras oficinas estatales a cargo de "asuntos de la mujer". Los decenios de 1980 y 1990 se identifican frecuentemente como portadores de un cambio en el activismo local y regional a través de la incorporación de los movimientos sociales a redes transnacionales. En este contexto, autoras como Sonia Álvarez (1999) han identificado el fenómeno de la ONGeización del activismo feminista.

Al mismo tiempo, estas fueron décadas de "tecnificación" en el manejo de la violencia contra las mujeres. La preocupación del Estado por proteger a la familia se renovó y se reconstruyó como un problema social a través de nuevos procesos de gubernamentalización basados en disciplinas técnicas que surgieron como respuestas a problemas de política pública y reforma legal. Aparecieron las primeras referencias a la violencia contra las mujeres como un obstáculo para el desarrollo económico y como una cuestión de salud pública. El Banco Mundial reconoció a la violencia contra las mujeres como un "problema de desarrollo relacionado con el género" y lo asoció con problemáticas de salud pública, como la prevalencia del alcoholismo en los varones (The World Bank, 2000). Así también, la Organización Panamericana de la Salud (OPS) identificó a la violencia doméstica como un factor de riesgo para las mujeres durante su ciclo reproductivo (Cuvi, 1999). Nuevamente, ahora través del lente técnico de las agencias transnacionales, la intervención en la violencia doméstica se (re)construyó como un objetivo gubernamental de optimización de la ciudadanía para alcanzar el desarrollo. Pero el abordaje de la violencia contra las mujeres como un problema de salud materna o como un obstáculo para el desarrollo económico, no necesariamente desafió los roles poscoloniales de género en su esencia. 
En 1993, durante la Conferencia de Viena sobre Derechos Humanos de las Naciones Unidas, se declaró que "los derechos de las mujeres son derechos humanos". Esto se reafirmó en la Cuarta Conferencia Mundial sobre la Mujer celebrada en Beijing en 1995. Su Plataforma de Acción identificó la violencia contra las mujeres como un "área de preocupación crítica" agravada por "la falta de leyes que prohíban efectivamente la violencia contra las mujeres; falta de reforma de las leyes vigentes; esfuerzos inadecuados por parte de las autoridades para promover el conocimiento y hacer cumplir las leyes existentes "(United Nations, 1995, párr. 118). El proceso de Beijing fue muy influyente en las redes feministas de América Latina. La Plataforma impulsó la creación de nueva legislación sobre violencia contra las mujeres en prácticamente todos los países de la región ( $\mathrm{Ma}-$ caulay, 2006). De esta manera, el lenguaje de los instrumentos transnacionales fue utilizado por organizaciones locales de mujeres, las cuales comenzaron a proyectar a la justicia penal como una herramienta que podría utilizarse para una variedad de efectos, incluida la lucha contra la violencia contra las mujeres.

En el contexto regional, la Organización de Estados Americanos (OEA) nombró en 1994 a su primer Relator Especial sobre los derechos de la mujer. En el mismo año, adoptó el primer tratado internacional especializado en violencia contra las mujeres en el mundo: la Convención para la Prevención, el Castigo y la Erradicación de la Violencia contra la Mujer, conocida como "Convención de Belem Do Pará", por la ciudad brasileña donde se suscribió. Belem do Pará se basa en la conceptualización de la violencia contra las mujeres como violación de los derechos humanos; su artículo 7, que establece los deberes que los Estados adquieren como compromisos urgentes, se enfoca principalmente en mecanismos de reforma legal. Seis de sus ocho puntos se refieren a la adopción de medidas legales, incluidas sanciones y reformas penales. Estos procesos colocaron a los Estados en la obligación de cumplir los compromisos adquiridos, pero las campañas internacionales aterrizaron en la legislación nacional de forma peculiar. Lo que identificaremos en este punto es un encuentro de discursos, entre los cuales tenemos las narrativas coloniales sobre la protección a la familia, los paradigmas internacionales de los de- rechos humanos y la institucionalización del género como una categoría de análisis impulsada por movimientos de mujeres agrupados en ONG y las nuevas agencias estatales.

Así, desde 1985, en Sao Paulo, Brasil, había iniciado una tendencia regional de creación de comisarías especializadas para mujeres, que precedieron a la promulgación de leyes nacionales sobre violencia contra las mujeres (Jubb, 2008). En el Ecuador, la Fundación María Guare envió a una de sus abogadas a Lima para averiguar cómo funcionaban las comisarías especializadas, con el objetivo de crear un modelo similar en el país (exfuncionaria de la Fundación, comunicación personal, abril 13, 2015). En 1994, se crearon las primeras "Comisarías de la mujer y la familia". Si bien fueron los movimientos de mujeres los que impulsaron la creación de estas comisarías, su configuración real no reflejó exactamente lo concebido por aquellos en términos de subordinación de género. Así, las comisarías se crearon sobre la premisa de que la familia, cuyos miembros vulnerables son las mujeres y los niños, necesitaba de protección para prevenir el abuso doméstico y sus consecuencias devastadoras para la salud pública y el desarrollo (Ministerio de Gobierno y Policía, 1994). El propio nombre de las agencias sugiere cuál fue su ámbito de acción planificado. De acuerdo con una profesional del derecho que trabajó en una ONG asociada con una comisaría, la expresión "y la familia" se agregó a la denominación por solicitud del Ministerio de Gobierno (comunicación personal, abril 13, 2015). De hecho, la exposición de motivos en el Acuerdo Ministerial que creó las comisarías indicaba que la "violencia contra las mujeres y los menores" era un problema social que necesitaba recursos para ser "manejado técnicamente" (Ministerio de Gobierno y Policía, 1994).

De cualquier manera, la idea de crear una ley especializada para hacer frente a la violencia contra las mujeres fue calando y en última instancia condujo en 1995 a la creación de la Ley 103, la primera en Ecuador sobre la violencia contra las mujeres y la familia. Si bien este cuerpo legal es un logro pionero de los movimientos de mujeres que lo impulsaron, en el texto final, la prevención y sanción de la "violencia familiar" se posicionaron como la ratio legis más esencial. Al título del proyecto, originalmente «Ley contra la violencia 
hacia las mujeres», se le agregó la expresión «y la familia» después de las negociaciones preliminares, al parecer porque una ley para la protección de la familia estaba mejor situada estratégicamente para ser aprobada por el Congreso Nacional. Otra vez, la protección a las mujeres se equiparó con la protección a la familia y se vinculó con la regulación de la domesticidad, sin un reconocimiento explícito de las "desigualdades en las relaciones de poder dentro de la pareja", que las feministas denunciaban (Camacho \& Hernández, 2011, pág. 237).

La armonía familiar fue, así, considerada el bien jurídico central por parte de varios legisladores: el diputado Santiago Bucaram, por ejemplo, solicitó a la Comisión de Asuntos Civiles y Penales presentar, además de la Ley 103, "un proyecto para que el Estado se preocupe por la situación de la familia [...], porque no solo se trata de dividir a la familia, sino también de defender a la familia "(Congreso Nacional del Ecuador, 1995, p. 78). La reinscripción de estas nociones fue evidente también en otros momentos: durante el segundo debate, el propio diputado Bucaram objetó que la ley se refiriera a la protección de la "libertad sexual" de las mujeres, e indicó que la expresión "integridad sexual" era más adecuada. En sus palabras: "no hay, en ninguna parte, una definición que nos diga de qué tipo de libertad sexual se habla" (Congreso Nacional del Ecuador, 1995, p.10). De acuerdo con el entonces diputado, hablar de "libertad sexual" equivalía a dejar abierta una puerta programática para legitimar actividades como la prostitución. Luego, otro diputado solicitó preservar el articulado original como un tributo a "estas hermosas mujeres" (quizá refiriéndose a las activistas que estaban presentes en la sesión), y para "salvarlas" después de "dos mil años de existencia cristiana" (Congreso Nacional del Ecuador, 1995, p.10). Estos ejemplos ilustran una continuidad de imaginarios en los que las mujeres son dignas de protección solo dentro del contexto familiar y siempre que se ajusten a los parámetros de moralidad prescritos por los paradigmas poscoloniales de la familia. Algunos ejemplos de modificaciones que se hicieron al proyecto de ley original, mirando más a la protección a la familia que a los derechos de las mujeres, pueden verse en la Tabla 1.

\section{Tabla 1. Reencuadre de conceptos sobre violencia familiar en la Ley 103}

\begin{tabular}{|c|c|c|}
\hline Proyecto de Ley & Discusión y debate & Conceptos aprobados \\
\hline Violencia contra las mujeres & Violencia contra la mujer y la familia & $\begin{array}{l}\text { Violencia contra la mujer y la } \\
\text { familia }\end{array}$ \\
\hline $\begin{array}{l}\text { El concepto de familia incluye } \\
\text { a la pareja de hecho }\end{array}$ & $\begin{array}{l}\text { La pareja de hecho no es miembro } \\
\text { de la familia }\end{array}$ & $\begin{array}{l}\text { La familia nuclear no incluye a } \\
\text { la pareja de hecho }\end{array}$ \\
\hline Libertad sexual & Integridad sexual & Libertad sexual de la familia \\
\hline $\begin{array}{l}\text { Designación de pago de } \\
\text { pensiones alimenticias como } \\
\text { medidas inmediatas tras de- } \\
\text { nuncia de violencia }\end{array}$ & $\begin{array}{l}\text { Pensiones alimenticias restringen } \\
\text { los derechos de los varones }\end{array}$ & $\begin{array}{l}\text { No se aprueba la pensión } \\
\text { alimenticia inmediata }\end{array}$ \\
\hline
\end{tabular}


Adicionalmente, es importante indicar que el movimiento de mujeres había concebido al proyecto de ley como una alternativa de justicia especializada. Así, el borrador era aplicable a los casos de violencia contra las mujeres que aún no constituían delito penal, es decir, la intención era llenar un vacío en la legislación para permitir el enjuiciamiento de problemas como abuso verbal, humillación, coerción sexual, hostilidad diaria, abuso emocional y lesiones físicas que no dejan huellas corporales. No obstante, el texto final adaptó las categorías de violencia (a excepción de la violencia psicológica) a las definiciones y procedimientos ya previstos como contravenciones en el Código Penal, pues la comisión legislativa encargada manifestó que una infracción de violencia familiar que no fuese al menos contravención era "inconcebible" (Congreso Nacional del Ecuador, 2004). Esto acabó por confinar a un cuerpo legal pensado como justicia especializada, a los marcos y limitaciones del aparato penal ordinario. La idea feminista de que la violencia contra las mujeres es diferente en su naturaleza a las ofensas comunes fue así marginada.

Se ha mostrado así, que los primeros giros punitivos hacia a la justicia penal en materia de violencia contra las mujeres estuvieron impulsados, por un lado, por la adopción de los marcos internacionales de derechos humanos y la tecnificación del manejo de la violencia contra las mujeres, pero, por otro lado, el giro solo fue posible porque los marcos de derechos humanos no amenazaron a los existentes discursos referenciales poscoloniales sobre la protección a la familia, y fue a través de estos últimos que la legislatura le dio sentido y significado a las propuestas de los movimientos de mujeres. Al mismo tiempo, fue el sistema penal nacional y no el marco especializado propuesto originalmente por las activistas, el que sirvió para canalizar y replantear el discurso feminista, siempre en la medida en la que se preservara la protección a la familia.

\section{5. Conclusiones: lecciones del siglo $\mathrm{XX}$ para una crítica del siglo XXI. Este artículo ha revelado las conexiones discursivas existentes entre las representaciones coloniales de la mujer y la familia, y las leyes y políticas públicas sobre violencia contra las mujeres que prosperaron en el siglo XX. Se ha evidenciado cómo los constructos de género, raza y clase que surgieron con la colonización, constituyeron ejes de las tecnologías gubernamentales que fueron adquiriendo caracteres adicionales a través del siglo XX. Así, a las leyes y políticas públicas existentes que protegían a la mujer en tanto madre de familia, se fueron sumando los discursos internacionales de los derechos humanos, y las nociones científicas que fueron definiendo a la violencia contra las mujeres como un problema de salud pública y de desarrollo.

La evidencia presentada en este artículo permite apreciar que la mujer que protege la ley del siglo XX es una que tiene tiempo, recursos, cumple un papel "probo" en la familia y, solo entonces, merece ser resguardada de un agresor que, a su vez, solo es censurado porque pone en peligro a la cohesión familiar. La política pública ecuatoriana del siglo XX refleja preocupación por tutelar los derechos de la mujer colonialmente construida, pero no muestra iniciativas para contrarrestar otras formas de violencia, como, por ejemplo, la violencia callejera contra las trabajadoras sexuales, la violencia sexual normalizada en las zonas rurales, el incesto, los crímenes de odio contra mujeres trans, etc. Se concluye, por lo tanto, que una de las razones por las que el proyecto de criminalizar la violencia doméstica contra las mujeres fue exitoso a mediados de los noventa, es que la estrategia penal encontró un terreno común con los discursos ya consolidados que reproducen una imagen poscolonial de la familia, la cual es el principal marco de inteligibilidad para justificar la protección legal de las mujeres.

Otro punto importante a tener en cuenta es que el lenguaje de los derechos humanos de las mujeres que emergió en los años noventa, y la tecnificación del tratamiento de la violencia doméstica, no desplazaron al discurso racializado y engenerizado de protección a la familia. Éste último ha podido incorporar y adaptar al lenguaje de los derechos humanos. Es decir, incluso el lenguaje de los derechos humanos puede ser una vía para legitimar la regulación de la familia de una manera aparentemente progresista, pero sin desafiar los discursos coloniales que limitan la vida de las mujeres en tanto miembros del núcleo familiar. 
Naturalmente, no se trata de sostener que los enfoques internacionales de derechos humanos son perjudiciales, ni es cuestión de negar que han sido históricamente transformadores. Más bien se evidencia la necesidad de ubicarlos en contexto, identificar qué elementos del discurso llegan a anclar en la regulación local, y qué papel cumplen respecto de la legitimación de las instituciones legales que marginan a las mujeres "desviadas", desde la perspectiva de la colonialidad del género. Esto permite, además tomar conciencia de las limitaciones de las estrategias legales a las que recurren los movimientos emancipatorios, y observar cómo los paradigmas globalizados pueden legitimar la expansión del control social, la perpetuación de la colonialidad, la invisibilización de la experiencia vivida por los cuerpos subordinados y, por lo tanto, la exclusión de quienes quedan fuera de las subjetividades legales predefinidas.

Arribando al siglo XXI, que transcurre, no son pocas las lecciones que nos ha dejado el siglo XX. La pasada centuria nos mostró lo que puede ocurrir cuando las demandas feministas se incorporan a la legislación a través de los discursos hegemónicos, mostrando que con frecuencia lo que abre el espacio para que las voces de las mujeres sean incorporadas al sistema legal es su adaptación a esquemas que ya se encuentran institucionalizados y que suelen ser, paradójicamente, aquello contra lo que los movimientos sociales luchan. Más específicamente, las narrativas poscoloniales de protección a la familia que se incrustaron en la Ley 103, no fueron perturbadas pese a los mejores esfuerzos de los movimientos de mujeres de ese entonces. Estas lecciones son útiles no solo para analizar cuerpos legales actuales, sino también para repensar estrategias por parte de los movimientos sociales que han visto en la reforma del derecho una herramienta para la emancipación.

Las construcciones poscoloniales del género y la raza continúan informando a las leyes. En particular, la protección del derecho a una vida libre de violencia se ha vinculado estrechamente a la penalización, casi como único camino posible. Esta estrategia enfatiza la responsabilidad individual, desplazando al análisis profundo de problemas sociales sistémicos, y vinculando a la violencia contra las mujeres con definiciones legales y técnicas que a su vez afectan a la forma en que los administradores de justicia pueden implementar las leyes (Tapia Tapia, 2016; Tapia Tapia, 2018). El espacio experiencial del proceso legal, entonces, se articula mediante conjuntos de prácticas formales que se basan en los conocimientos dominantes y las narrativas poscoloniales, determinando las maneras en que las mujeres pueden considerarse a sí mismas sujetos legales, cómo son vistas por los jueces y las defensas técnicas, y hasta qué punto son consideradas dignas de protección legal. El feminismo transnacional ha promovido un discurso basado en derechos, pero en un sitio poscolonial como el Ecuador, éste ha sido acogido solo cuando la colonialidad ha sido menos desafiada.

Estos hallazgos invitan a más preguntas y más investigaciones para quienes interrogan el uso de la justicia penal en la lucha por la erradicación de la violencia contra las mujeres. Debemos reconocer a la colonialidad del género como un límite epistémico del discurso jurídico contemporáneo. Las narraciones contrahegemónicas pueden empujar al límite a las epistemes dominantes, pero no están fuera de ellas. Por eso es necesario continuar interrogando a los principios más universalizados del derecho, a las políticas públicas de protección a la familia, y también a los marcos internacionales de derechos humanos. Esto permite identificar cuáles son las voces silenciadas y excluidas. La ley delimita espacios para la normalización de los cuerpos femeninos, pero cuando estos caen fuera de los paradigmas dominantes, el derecho puede propiciar su aniquilación. Las formas cómo las mujeres experimentan la ley y lo que les permite imaginar también deben informar al derecho. Tenemos que explorar el pluralismo jurídico y la resistencia política en los límites mismos de lo que es inteligible y de lo que (aún) no es posible articular con el lenguaje jurídico.

\section{6.}

\section{Referencias}

Álvarez, S. E. (1999). Advocating feminism: The Latin American Feminist NGO <Boom $>$. International Feminist Journal of Politics, 1(2), 181209. doi:10.1080/146167499359880

Bacchi, C. L. (1999). Women, policy and politics: The construction of policy problems. London: Sage.

Bacchi, C. L. (2012). Why study problematisa- 
tions? Making politics visible. Open Journal of Political Science, 2(1), 1-8.

Bernstein, E. (2012). Carceral politics as gender justice? The "traffic in women" and neoliberal circuits of crime, sex, and rights. Theory and Society, 41(3), 233-259.

Briones, M. (1988, March). Ciudadanas de segunda clase. Matapalo, 12, 4.

Bumiller, K. (2008). In an abusive state: how neoliberalism appropriated the feminist movement against sexual violence. Durham and London: Duke University Press.

Camacho, G., \& Hernández, K. (2011). Comisarías de la mujer y acceso a la justicia en Cuenca. In L. Pásara (Ed.), El funcionamiento de la justicia del Estado (pp. 229-282). Quito, Ecuador: Ministerio de Justicia y Derechos Humanos, Naciones Unidas.

Clark, K. (2001). Género, raza y nación: La protección a la infancia en el Ecuador (1910 1945). In G. Herrera (Ed.), Antología género (pp. 9). Quito: FLACSO Ecuador.

Código penal, Publicación oficial (1938). Recuperado de Fiel Web https://www.fielweb.com

Congreso Nacional del Ecuador. (1995, noviembre 14). Acta No. ocho.

Congreso Nacional del Ecuador. (2004, octubre 13). Acta No. 24-304.

Corrigan, R. (2006). Making meaning of Megan's Law. Law \& Social Inquiry, 31(2), 267-312.

Cuvi, M. (1999). Violencia contra las mujeres. La ruta crítica en el Ecuador. Quito: Organización Panamericana de la Salud.

Díaz, A. (2001). Women, order and progress in Guzmán Blanco's Venezuela, 1870 - 1888. In R. Salvatore, C. Aguirre \& J. Guilbert (Eds.), Crime and punishment in Latin America: law and society since late colonial times (). Durham and London: Duke University Press.
Dore, E. (2000). Property, households and public regulation of domestic life. Diriomo, Nicaragua, 1840-1900. In E. Dore, \& M. Molyneux (Eds.), Hidden histories of gender and the state in Latin America (pp. 3). Durham and London: Duke University Press.

Dore, E., \& Molyneux, M. (Eds.). (2000). Hidden histories of gender and the state in Latin America. USA: Duke University Press.

Foucault, M. (1982). The subject and power. Critical Inquiry, 8(4), 777-795.

Foucault, M. (1991). In Burchell G., Gordon C. and Miller P. (Eds.), The Foucault effect: Studies in governmentality. Chicago: University of Chicago Press.

Gargallo, F. (2010). Una metodología para detectar lo que de hegemónico ha recogido el feminismo académico latinoamericano y caribeño. En N. Blazquez Graf, F. Flores Palacios \& M. Ríos Everardo (Eds.), Investigación feminista. Epistemología, metodología y representaciones sociales (pp. 155). México: Centro Regional de Investigaciones Multidisciplinarias, UNAM.

Gargallo, F. (2014). Feminismos desde Abya Yala. Ideas y proposiciones de las mujeres de 607 pueblos en Nuestra América. Ciudad de México: Editorial Corte y Confección.

Golder, B., \& Fitzpatrick, P. (2009). Foucault's law. USA and Canada: Routledge.

Gotell, L. (1998). A critical look at state discourse on "violence against women": some implications for feminist politics and women's citizenship. Women and Political Representation in Canada (pp. 39) University of Ottawa Press.

Guy, D. J. (2000). White slavery and mothers alive and dead: the troubled meeting of sex, gender, public health, and progress in Latin America. United States: University of Nebraska Press.

Halley, J., Kotiswaran, P., Shamir, H., \& Rebouché, R. (2018). Governance feminism: an introduction. Minneapolis and London: University of Minnesota Press. 
Halley, J., Kotiswaran, P., Shamir, H., \& Thomas, C. (2006). From the international to the local in feminist legal responses to rape, prostitution/sex work, and sex trafficking: Four studies in contemporary governance feminism. Harvard Journal of Law \& Gender, 29, 335-423.

Htun, M. (2003). Sex and the state: abortion, divor$c e$, and the family under Latin American dictatorships and democracies. USA: Cambridge University Press.

Jubb, N. (2008). Mapeo regional de las comisarías de la mujer en América Latina. Quito: CEPLAES.

Lind, A. (2012). "Revolution with a Woman's face"? Family, norms, constitutional reform, and the politics of redistribution in post neoliberal Ecuador. Rethinking Marxism: A Journal of Economics, Culture \& Society, 24(4), 536-555.

Lugones, M. (2010). Toward a Decolonial Feminism. Hypatia, 25(4), 742-759. doi:10.1111/ j.1527-2001.2010.01137.x

Macaulay, F. (2006). Judicialising and (de) criminalising domestic violence in Latin America. Social Policy and Society, 5(1), 103.

Mignolo, W. (2011). The darker side of western modernity. Global futures, decolonial options. Durham \& London: Duke University Press.

Ministerio de Gobierno y Policía. (1994). Disposiciones organizativas para el funcionamiento de las comisarías de la mujer y la familia. Acuerdo No. 3548: Registro Oficial No. 410.

Oksala, J. (2013). Feminism and neoliberal governmentality. Foucault Studies, (16), 32-53.

Padrón, T. (2019). Las instituciones del sistema moderno-colonial de género y su materialización en la primera constitución del Ecuador. (Unpublished Master's degree dissertation). Universidad de Cuenca.

Quijano, A. (2000). Colonialidad del poder y clasificación social. Journal of World-Systems Research, XI(2), 342-386.
Radcliffe, S. A., \& Westwood, S. (1996). Remaking the nation: place, identity and politics in $\mathrm{La}^{-}$ tin America. London and New York: Routledge.

Rendón, Z. (2006). Cómo se juzga al feminismo verdadero. Orígenes del feminismo en el Ecuador. (pp. 93). Quito: CONAMU, FLACSO Sede Ecuador, Comisión de género y equidad social del MDMQ, UNIFEM.

Rodríguez, E. (2000). Civilizing Domestic Life in the central valley of Costa Rica, 1750-1850. In E. Dore, \& M. Molyneux (Eds.), Hidden histories of gender and the state in Latin America (pp. 85-107). Durham and London: Duke University Press.

Shore, C., \& Wright, S. (1997). Anthropology of policy: perspectives on governance and power. London and New York: Routledge.

Smart, C. (1989). Feminism and the power of law. London: Routledge.

Sobrevilla, D. (2008). La filosofía andina del P. Josef Estermann. Solar, 4(4), 231-247.

Stepan, N. (1991). The hour of eugenics: race, gender, and nation in Latin America. United States: Cornell University Press.

Stoler, A. L. (1995). Race and the education of desire: Foucault's history of sexuality and the colonial order of things. USA: Duke University Press.

Tapia Tapia, S. (2016). Sumak Kawsay, coloniality and the criminalisation of violence against women in Ecuador. Feminist Theory, 17(2), 141156.

Tapia Tapia, S. (2018). Feminism and penal expansion: the role of rights-based criminal law in post-neoliberal Ecuador. Feminist Legal Studies, 26(3), 285-306. doi:https://doi. org/10.1007/s10691-018-9380-5

The World Bank. (2000). Ecuador gender review. Issues and recommendations. Washington: The World Bank. 
Tinsman, H. (2001). Good Wives and Unfaithful Men: Gender Negotiations and Sexual Conflicts in the Chilean Agrarian Reform, 19641973. Hispanic American Historical Review, 81(3), 587-619.

Tinsman, H. (2002). Partners in conflict: The politics of gender, sexuality, and labor in the Chilean agrarian reform, 1950-1973. United States: Duke University Press.

UNESCO. (1984). Problems that concern women and their consideration in development planning. (Report presented to UNESCO by the Centro Ecuatoriano de Investigación Social). Quito: UNESCO. Retrieved from http://unesdoc. unesco.org/ Retrieved from http://unesdoc. unesco.org/images/0006/000632/063207eb. pdf

United Nations. (1995). Beijing declaration and platform for action. Retrieved from http:// www.un.org/esa/gopher-data/conf/fwcw/ off/a--20.en 